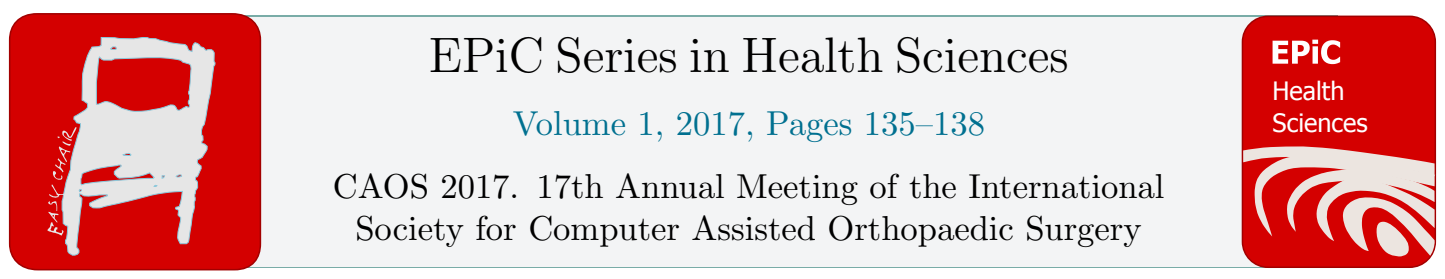

\title{
Development of a Knee Physical Exam Haptic Training Simulator: Validation of the Computational Haptic Environment

\author{
Joshua W Giles $\mathrm{PhD}^{1 * * \dagger}$, Yawen Chen MSc, Stuart Bowyer PhD ${ }^{* 2}$ \\ ${ }^{1}$ Mechatronic in Medicine Lab, Imperial College London, U.K. \\ ${ }^{2}$ Wearable Technologies Lab, Imperial College London, U.K. \\ joshua.giles@imperial.ac.uk
}

\begin{abstract}
This paper presents the development and validation of an early computational model intended to drive a haptic simulator for knee physical exam skills training. Specifically, an analytical model of knee soft tissues was developed and validated against existing computationally expensive open source software and through cadaveric experimentation.
\end{abstract}

\section{Introduction}

One of an orthopaedic surgeon's fundamental skills is their ability to assess a patient's joints effectively and accurately through manual physical exam techniques. Becoming proficient in these skills is difficult due to the wide range of joint conditions that a surgeon must be able to differentiate and the limited practice opportunities. Coles et al. (2011) found that a range of medical training challenges had been addressed using haptic simulators. However, to our knowledge, all past orthopaedic haptic simulators have focused on mimicking the interaction of a surgeon held tool with anatomy, rather than the surgeon directly interacting with a simulated patient. With this in mind, we have begun to develop a haptic simulator to aid in knee physical exam training.

This haptic training simulator has three parts (Figure 1): a computational knee model to simulate the kinetic environment, a haptic device for surgeon interaction, and a control program. The final physical simulator is made of a phantom knee model with the tibia connected to the haptic device and the femur fixed; however, the articular surfaces and soft tissues (e.g. ligaments) are omitted so that the physical model is non-force producing. The surgeon grasps the tibia model, as they would for a physical exam, and applies motions matching an exam task. The 6 degree-of-freedom (DOF) pose of the haptic device during exam motions is recorded, transformed to an anatomical knee coordinate system after which knee joint and haptic loads are calculated and fed back to the surgeon through the haptic device.

In recently presented work (Chen et al. 2016), we described the development of a computational biomechanics knee model - based on the open-source OpenSim software (Delp et al. 2007) - that could

\footnotetext{
${ }^{*}$ These two authors are considered co-senior authors
} 
replicate the knee's kinetic response to simulated physical exam tasks (e.g. draw test). However, this work concluded that the OpenSim-based model could not produce a sufficiently fast operation rate to provide high quality haptic rendering. As such, the current work sought to develop and validate a custom Matlab-based numerical knee model that could accurately calculate knee kinetics at a rate of $\sim 1 \mathrm{kHz}$ (Salisbury et al. 2004). Once developed, the model was validated against the OpenSim software and experimental data.

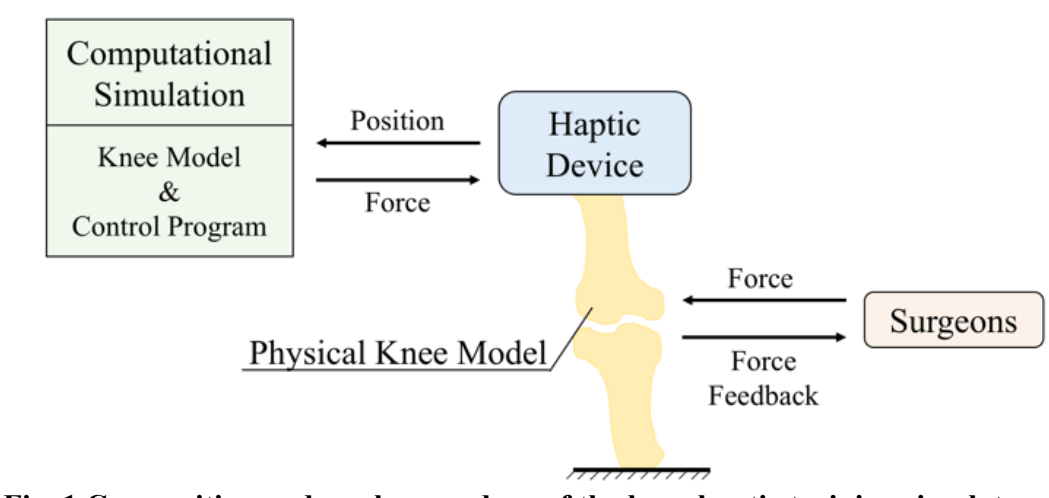

Fig. 1 Composition and work procedure of the knee haptic training simulator.

\section{Materials \& Methods}

\section{Numerical Model}

The inability of the OpenSim-based knee model to achieve sufficient haptic rendering is attributable to the complex iterative optimization used to achieve accurate results during dynamic motions. However, clinical physical exam tasks are quasi-static and thus, iterative optimization is not warranted. Therefore, we developed a Matlab-based numerical model of the knee with its geometric and material (e.g. tissue stiffness) properties drawn from a previous OpenSim model (Xu et al. 2015).

The new model permits 6 DOF motion and considers the geometry of the femur, tibia, and patella. Knee muscle forces were assumed to be zero because physical exams are conducted with patients in a relaxed position. Load is produced by the tibial gravity force and four knee ligament groups: ACL, PCL, MCL, \& LCL. These ligaments were further split into ten bundles with unique lines of action, attachments, resting lengths, and stiffnesses to improve the model's physiologic accuracy.

The surgeon's interaction with the numerical model was modelled as a 3 DOF force and 3 DOF moment applied with a clinically standard hand position.

\section{Validation against OpenSim}

We first investigated the internal validity of our load calculation algorithm by comparing the haptic loading results for two clinical exam motions to the results produce using the widely accepted OpenSim software. This comparison was expected to produce near identical results because the parameters that define our Matlab model were drawn from Xu's existing OpenSim model. The two tested motions were anteroposterior translation (i.e. the clinical AP draw test) and adduction-abduction rotation (i.e. the clinical varus-valgus laxity test). In addition, the new model's run time performance was assessed.

\section{Cadaveric Validation}

Subsequently, we assessed the model's external validity - its ability to produce objectively accurate, physiological results - by using a validated cadaveric-robotic testing system (Athwal et al. 2016) that 
can accurately perform the two clinical tests described in the above section. One knee specimen was tested and, in addition to motion trials, anatomical measurements were made to enable the Matlab model to be scaled to match the tested specimen.

\section{Results}

Results from the validation against OpenSim demonstrated a close agreement of our model for both AP draw tests (\%RMSE: $0.071 \pm 0.065 \%$ for force \& $0.071 \pm 0.069 \%$ for moments) and varus-valgus rotation tests (\%RMSE: $0.72 \pm 0.52 \%$ for force \& $5.51 \pm 5.63 \%$ for moments). Despite the markedly higher RMS error for moments in the varus-valgus test, this still only corresponds to an absolute difference of $0.01 \mathrm{Nm}$. With respect to runtime performance, our model executed with a speed of 0.00087 seconds on average in comparison to 0.188 seconds for the comparable OpenSim model.
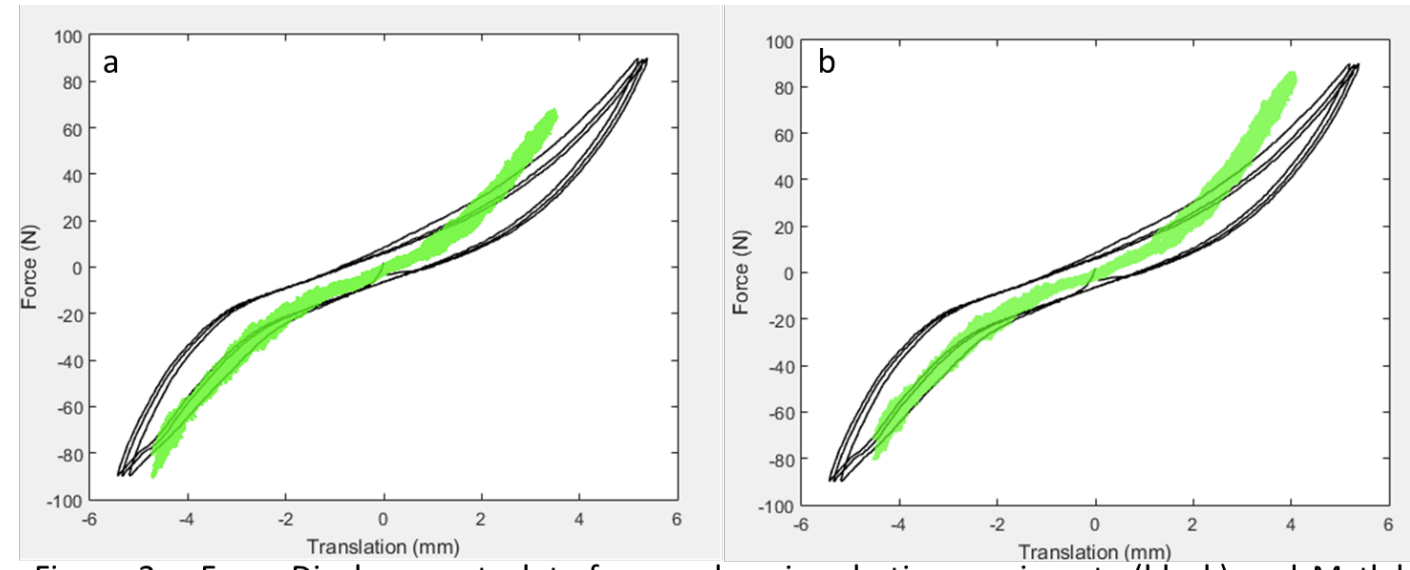

Figure 2 - Force-Displacement plots from cadaveric robotic experiments (black) and Matlab knee model simulations (green) for an AP Drawer test. (a) Simulation results using generic ligament stiffness values, and (b) simulation results after scaling ligament stiffness.

Experimental validation of our model at first demonstrated similar force-motion pathways to the robotic tests (Figure 2a) but differing magnitudes. This discrepancy was expected as the experiment could not provide cadaveric ligament stiffness values to be input into our model. However, after adjusting the ligaments' stiffnesses, based on biomechanical principles, the plots were accurately matched (Figure 2b).

\section{Discussion}

The goal of this work was to develop and validate a numerical knee biomechanics model that can accurately replicate quasi-static knee motion-loading relationships with sufficient temporal resolution to provide high quality haptic rendering. The results of this work have demonstrated that our model could achieve a refresh rate of up to $1.15 \mathrm{kHz}$ which exceeds the accepted limit of $1 \mathrm{kHz}$ for good haptic rendering (Salisbury et al. 2004). As well, our two validations have shown the internal and external validity of the model through its ability to replicate the results of the widely used OpenSim software (Delp et al. 2007) and to produce physiologically accurate results that agree with the findings of a cadaveric trial. Initial haptic trials with a Phantom Omni device (Sensable Technologies) are ongoing 
and in the future this model will be implemented for use with a Kuka lightweight robot built for human interaction.

\section{References}

Athwal K.K., El Daou H., Lord B., et al. (Advanced Online Publication) Lateral soft-tissue structures contribute to cruciate-retaining total knee arthroplasty stability. Journal of Orthopaedic Research; 2016; 10.1002/jor.23477.

Chen Y., Bowyer S.A., Giles J.W. Development of a Haptic Simulator for the Training of Surgeons in Knee Physical Exam Skills. International Conference on Computer and Robot Assisted Surgery. 2016.

Coles T.R., Meglan D., John, N.W.. The role of haptics in medical training simulators: A survey of the state of the art. IEEE Transactions on Haptics; 2011; 4(1):51-66.

Delp S.L., Anderson F.C., Arnold A.S., et al. OpenSim: Open-source software to create and analyse dynamic simulations of movement. IEEE Transactions on Biomedical Engineering; 2007; 54(11):19401950.

Salisbury K., Conti F., Barbagli F. Haptic rendering: introductory concepts. IEEE Computer Graphics and Applications; 2004; 24(2):24-32.

Xu H., Bloswick D., Merryweather A. An improved OpenSim gait model with multiple DOF knee joint and knee ligaments. Computer Methods in Biomechanics and Biomedical Engineering; 2015; 18(11): 1217-1224. 